Jörg Huffschmid, Elmar Altvater

\title{
Ein Gespräch über »politischen Kapitalismus«, »Stamokap«, Wettbewerbsfähigkeit und vieles andere
}

Vorbemerkung: In der letzten Nummer der PROKLA (Europa I: Osteuropa und der Westen, September 1998) ist von Melanie Tatur und Jadwiga Staniszkis der Begriff »Politischer Kapitalismus« benutzt worden, um die Gesellschaftsformation theoretisch zu fassen, die sich in einigen osteuropäischen Ländern derzeit herausbildet. Unmittelbar nach den samtenen Revolutionen der späten 80er und frühen 90er Jahre herrschte in den Sozialwissenschaften die Auffassung vor, es handle sich bei den durch die Revolutionen geöffneten Entwicklungswegen um Pfade der Modernisierung, an deren Ende das verheißungsvolle Bild einer effizienten Marktwirtschaft, einer pluralistischen Zivilgesellschaft und parlamentarischen Demokratie zu sehen war. Inzwischen aber sind sich selbst Modernisierungstheoretiker klar darüber, daß dieses Bild zumindest in den unterstellten Fristen eine fata morgana gewesen ist. In Mittel- und Osteuropa bilden sich offenbar kapitalistische Hybridformen heraus mit Eigentumsformen, Regelungsmechanismen und Mischungsverhältnissen von Ökonomie und Politik, legitimierten und nicht-legitimierten vor- und außerkonstituionellen Mächten, die keinen Kapitalismus mit OECD-Profil entstehen lassen - sondern eben einen »politischen Kapitalismus«, wie neben Tatur und Staniszkis auch David Stark, Burawoy und andere hervorheben. Offensichtlich sind die Prozesse der Transformation nach der samtenen Revolution, die ja nur die politischen Herrschaftsverhältnisse über den Haufen geworfen, nicht aber sogleich in Gestalt eines big bang auch passende ökonomische und soziale Strukturen hervorgebracht haben, abhängig von den Raum- und Zeit-Koordinaten, in denen sie ablaufen. Die Beschreibung des Politischen Kapitalismus in Mittel- und Osteuropa erinnert, so neu einzelne Elemente der kapitalistischen Hybridform auch sind, in mancher Hinsicht an den »Stamokap«, den »Staatsmonopolistischen Kapitalismus«. Die Theorie des Staatsmonopolistischen Kapitalismus läßt sich bis auf Lenin, Eugen Varga und andere im ersten Viertel dieses Jahrhunderts zurückführen. Sie wurde nach dem Zweiten Weltkrieg in den real-sozialistischen Ländern zur quasi-offiziellen Theorie des modernen Kapitalismus. Diese Theorie wurde aber auch in der westlichen Welt aufgegriffen und fortentwickelt. In den 70er Jahren kulminierten die Auseinandersetzungen um den Stamokap, nicht zuletzt weil aus dieser Theorie bestimmte politische Bündniskonstellationen folgten, die zumindest in der westeuropäischen Linken außerordentlich kontrovers diskutiert wurden. Die - scheinbare oder tatsächliche Nähe der Konzepte des politischen Kapitalismus und des staatsmonopolistischen 
Kapitalismus haben uns dazu veranlaßt, mit Jörg Huffschmid ein Gespräch zu führen. Er war in den 70er Jahren einer der wichtigsten Vertreter einer westlichen Variante der Stamokap-Theorie. Die Fragen zielen auf die Berührungspunkte zwischen den Theorien von Stamokap und Politischem Kapitalismus, aber auch darauf, was von der Stamokap-Theorie nach dem Ende des real existierenden Sozialismus im real existierenden Kapitalismus noch bleibt. Das Gespräch führte für die PROKLARedaktion Elmar Altvater.

E.A.: In dem im September erschienenen Europaheft der PROKLA schreibt Melanie Tatur in ihrem Aufsatz zum Politischen Kapitalismus: »In der Diskussion um die aktuelle und künftige Rolle des Staates hat sich seit den 80er Jahren ein Paradigmenwechsel vollzogen. Dem liegt die Einsicht zugrunde, daß mit der Internationalisierung der Kapitalmärkte und der Globalisierung des Wettbewerbs die Voraussetzungen für eine nationalstaatliche Wirtschaftspolitik im Sinne keynesianischer Konjunktursteuerung entfallen und die autonomen Handlungsmöglichkeiten auf staatlicher Ebene zunehmend eingeschränkt werden.« Jörg, würdest Du diese Vorstellung eines Paradigmenwechsels hinsichtlich der Rolle des Staates teilen?

J.H.: Ich würde sie nur sehr eingeschränkt teilen. Zwar ist die Autonomie des Nationalstaats gegenwärtig stärker eingeschränkt als früher; nationalstaatliche Politik und Gesetze müssen mehr auf die internationalen Bedingungen Rücksicht nehmen. Andererseits aber ist der Nationalstaat für die Organisation der materiellen Reproduktion und für den Lebenszuschnitt der allermeisten Menschen doch nach wie vor eine ganz entscheidende Bezugsgröße, die von den Menschen auch in erheblichem Maße beeinflußt und gestaltet werden kann. Die meisten Gesetze und die meisten Regulationsformen, die die Menschen konkret betreffen, sind nach wie vor sehr stark von nationalstaatlichen Einflüssen geprägt.

E.A.: Nun könnte man ja mit der Theorie (sofern es denn eine ist) des "politischen Kapitalismus « sagen: zumindest muß der Nationalstaat dafür sorgen, daß in der Marktwirtschaft entsprechende private Eigentumsrechte hergestellt werden. Dies ist auch die These von Tatur und Staniszkis in dem schon erwähnten PROKLA-Heft. Aber über diese Herstellung von Eigentumsrechten hinaus spielt der Nationalstaat in der globalisierten Ökonomie eine mindere Rolle als je zuvor. Diese Auffassung würdest Du nicht teilen? J.H.: Nein, diese Auffassung teile ich nicht. Sie erinnert mich vielmehr an eine recht krude Version der Theorie des »staatsmonopolistischen Kapitalismus«, wie sie vielleicht in den 60er und 70er Jahren vertreten wurde. Danach bestand die Rolle des Staates ausschließlich in der Erfüllung von Kapitalfunktionen und in sonst nichts. Ich denke, dies ist weder empirisch zutreffend noch theoretisch haltbar, weil die politischen Handlungsräume dabei einfach wegdefiniert worden sind. Die Frage nach der Rolle sozialer 
und politischer Bewegungen, nach einem eigenständigen Raum im Staat, in dem Politik eben nicht nur als Agent der Kapitalverwertung funktioniert, wird dabei gar nicht untersucht, sondern deduktiv verneint.

E.A.: Nun könnte man dem entgegenhalten, daß es den beiden PROKLAAutorinnen vor allem um die Transformationsprozesse in Osteuropa geht. Die Herstellung von privaten Eigentumsrechten ist sozusagen die Grundlage dafür, daß überhaupt Kapital entsteht, und daß mit dem Kapital, das da entsteht, auch Akkumulation, Wachstum und somit auch die höchst ersehnte Steigerung des Wohlstands zustandekommen; siehe Douglass North und andere. Dann stellt es sich aber bei näherer Betrachtung heraus, daß die Errichtung von Eigentumsrechten eine etwas kompliziertere Angelegenheit ist, als man angenommen hat, daß da Hybridformen entstehen, Clanbeziehungen eine Rolle spielen, paternalistische Beziehungen wichtig sind, Mafia-ähnliche Strukturen eine Rolle spielen und, und, und... Private Eigentumsrechte herzustellen ist zwar eine gute Idee, die aber schwer durchzuführen ist. Dieser Aspekt würde mit der Stamokap-Theorie nicht ohne weiteres zu erfassen sein, oder doch?

J.H.: Nein, die Theorie des staatsmonopolistischen Kapitalismus ist dazu wenig geeignet. Sie bezieht sich auf einen ganz anderen Sachverhalt, nämlich auf den entwickelten, ja hochentwickelten Kapitalismus. Sie untersucht, wie sich der moderne Staat zu den bereits ausgereiften Kapitalformen verhält. In Osteuropa handelt es sich im Unterschied dazu ja eher um Prozesse, die man vielleicht etwas übertrieben - mit dem Prozeß der »ursprünglichen Akkumulation«, also mit der Herausbildung von Eigentumsund Kapitalstrukturen vergleichen könnte. Dieses Beispiel hinkt natürlich, weil es zumindest einen eklatanten Unterschied zwischen der Transformation in Osteuropa und der ursprünglichen Akkumulation gibt: die Transformationsprozesse finden in einer ansonsten bereits kapitalistisch entwickelten Welt statt, was ja bei der ursprünglichen Akkumulation nicht der Fall war.

E.A.: Also geht es um die Frage der Ungleichzeitigkeit und der Ungleichmäßigkeit der Entwicklung, die jetzt bei der Untersuchung von Transformationsprozessen an zentraler Stelle berücksichtigt werden müßte. Mit anderen Worten, Zeit und Raum der Transformation spielen eine entscheidende Rolle, so daß ein zeitlos gedachter Transformationsprozeß theoretisch nichts herzugeben vermag. Vielleicht sollten wir an dieser Stelle dann aber fragen, was denn eigentlich die Kernelemente der Stamokap-Theorie sind, um vielleicht von da aus noch einmal die Frage aufzuwerfen, ob es zur Theorie des politischen Kapitalismus - sofern es eine ist - bestimmte Entsprechungen gibt.

J.H.: Aus meiner Sicht gibt es drei zentrale Elemente der traditionellen und auch der verfeinerten Theorie des staatsmonopolistischen Kapitalismus. 
Das erste ist die Annahme eines gesetzmäßigen Fortschreitens des Kapitalismus bis zu seiner Überwindung und der Einmündung in den Sozialismus. Dies ist so, wie sich die Theorie des staatsmonopolistischen Kapitalismus das vorgestellt hat, bekanntlich nicht eingetreten. Die These ist von der Geschichte widerlegt und taugt also auch nicht dazu, weltgeschichtliche Prognosen zu machen. Das zweite Element ist die Theorie des Monopols, also der Herausbildung einer durch Konzentration und Zentralisation beförderten systematisch differenzierten Kapitalstruktur, die durch das große Gewicht weniger großer Kapitale im gesamtwirtschaftlichen Reproduktionsprozess gekennzeichnet ist. Dies führt dazu, daß Macht und zwar ökonomische und außerökonomische Macht in der Konkurrenz eine große und weder in der Vorstellung von der unsichtbaren Hand bei Smith noch in der Konkurrenzkonzeption bei Marx vorgesehene Rolle spielt. Ich denke, das ist ein Theorieelement des staatsmonopolistischen Kapitalismus, das nach wie vor Bestand hat, auch wenn beispielsweise die Rolle kleinerer und mittlerer Unternehmen durchaus differenziert betrachtet werden muß. Das dritte Element, das das Staatsmonopolistische am Stamokap ausmacht, ist die Interaktion, Kooperation und Verflechtung des Staatsapparates mit den Monopolen als den maßgeblichen Strukturelementen und Akteuren der Wirtschaft. Dieses Element finde ich das Interessanteste an der Theorie des staatsmonopolistischen Kapitalismus. Hierbei hat es in den 70er und 80er Jahren erhebliche Weiterentwicklungen der Theorie gegeben. Diese bestehen im wesentlichen darin, daß die frühe, insbesondere in der Sowjetunion sehr stark unter politischen Gesichtspunkten entwickelte These, daß der Staat ausschließlich die Agentur der entwickelten Monopolkapitale ist, weitgehend aufgegeben werden mußte und dem Staat eine sehr viel komplexere Regulierungsfunktion zugesprochen wurde, deren Beeinflussung und Gestaltung zu unterschiedlichen Entwicklungs- und Bewegungsformen des Kapitalismus führt. In diesem Zusammenhang möchte ich auf theoretische Entwicklungen in Frankreich, die mit dem Namen Paul Boccara verbunden sind, aber auch in Westdeutschland im Umkreis des IMSF (Institut für Marxistische Studien und Forschung) und in der ehemaligen DDR vor allem im IPW (Institut für Politik und Wirtschaft) hinweisen. Sie alle haben zu einer Öffnung der Stamokap-Theorie geführt. Sie nahm Bewegungen zur Kenntnis, die nicht ausschließlich als Ausdruck der Verwertungslogik des Kapitals oder aus dem Widerspruch gegen die Verwertung des Kapitals erklärt werden konnten, also nicht auf den Gegensatz von Arbeit und Kapital reduzierbar waren. Diese Wandlung und Erweiterung der Theorie finde ich sehr wichtig, weil sich daraus meines Erachtens Berührungspunkte mit anderen Theorieansätzen, etwa mit der Regulationstheorie in Frankreich, oder mit dem in den USA etwa gleichzeitig entwickelten »social structure of accumulation«-Ansatz ergeben. Zwischen diesen drei theoretischen 
Entwicklungen gibt es Parallelen, die ich außerordentlich interessant finde, zumal sie unabhängig voneinander in verschiedenen Diskurszusammenhängen entwickelt worden sind.

E.A.: Danach wollte ich sowieso fragen, weil mir tatsächlich die - methodische und auch von den theoretischen Konzepten her - Nähe zur Regulationstheorie und zum »social structure of accumulation«-Ansatz, also zu den Theorieansätze der radicals in den USA, sehr wichtig erscheint. Aber bevor wir dazu kommen, möchte ich eine andere Frage noch einmal aufwerfen. Diese hat erneut mit Staat und Ökonomie und mit dem Paradigmenwechsel zu tun, von dem Melanie Tatur gesprochen hat. Die Globalisierung ist der Auslöser des Paradigmenwechsels, weil der Staat eine geringere Rolle auf globalen Märkten spielt. Erste Frage: Würdest Du dem zustimmen, oder siehst Du das anders? Die zweite Frage, die damit zu tun hat, betrifft das Verhältnis von Staat und großen Konzernen. Heute gibt es ja Konzepte, und auch das ist Teil des Paradigmenwechsels, von dem Tatur spricht, die nicht so sehr die Verflechtung zwischen Staat und großen Unternehmen ins Zentrum stellen, sondern den Staat als Moderator von Standortpolitik und Wettbewerbsfähigkeit im globalen Konkurrenzkampf, als Wettbewerbstaat also, analysieren und kritisieren. Bei der Frage nach den Bedingungen der Wettbewerbsfähigkeit am sogenannten Standort spielen natürlich nicht nur große Konzerne, sondern auch kleine und mittlere Betriebe und natürlich die Zivilgesellschaft und ihre Institutionen, die neuen und alten Bewegungen und Organisationen. eine große Rolle. Würdest Du das nicht vielleicht doch als eine Art Paradigmenwechsel sehen? Und wäre ein solcher Ansatz in die Stamokap-Theorie integrierbar?

J.H.: Ich bin vorsichtig mit dem Begriff Paradigmenwechsel. Die empirischen Beobachtungen, die Du anführst, können wir theoretisch durchaus ohne die Annahme eines Paradigmenwechsels im Rahmen der marxistischen Theorie erfassen. Der Staat ist ja auch in seiner Funktion als Garant der kapitalistischen Verwertungsbedingungen auf dem Weltmarkt nie unmittelbar als ökonomischer Organisator aufgetreten, war und ist als politische Hintergrundinstanz allerdings immer präsent z. B. als Handelsstaat oder als Militärmacht. Diese Rolle hat der Staat als imperialistischer Staat auch Anfang dieses Jahrhunderts schon gespielt, nicht als mit dem Kapital verflochtener Komplex, sondern als politische Macht hinter dem Kapital, die je nach den historischen Umständen unterschiedlich aggressiv ist. Insofern denke ich, ist da eigentlich soviel Paradigmenwechsel nicht zu sehen. Auch bei der Rolle des Staates als Garant der Kapitalverwertung ist jeweils der Zwischenraum zwischen dieser ganz allgemeinen Funktion und der unmittelbaren Indienstnahme durch einzelne beherrschende Kapitale wichtig. In diesem Zwischenraum haben kleine und mittlere Unternehmen und sonstige Akteure gewisse Handlungsfreiheiten, deren Umfang von der 
Entwicklung der politischen Strukturen oder der bürgerlichen Demokratie abhängt. Die politische Demokratie hat sich aber nicht unmittelbar als zwangsläufige Folge oder Begleitumstand des Kapitalismus, sondern parallel zum Kapitalismus herausgebildet. Daher ist sie auch nicht einfach als Funktion des Kapitals zu verstehen. Die Verflechtung dieser beiden Staatsebenen - der Staat als Regulationsinstanz der Kapitalverwertung, bei der die großen Kapitale eine maßgebliche Rolle spielen, da sie entsprechenden Druck auszuüben vermögen, und der Staat als Raum für die Entfaltung von bürgerlicher Demokratie und sozialer Bewegung, die sich nicht einfach auf Kapitalfunktionen reduzieren läßt, ist m.E. ein äußerst wichtiger und interessanter Gegenstand theoretischer wie empirischer Forschung. Dieser Aspekt war in der Theorie des staatsmonopolistischen Kapitalismus nicht enthalten. Spielräume wurden zwar gesehen, aber sie wurden voreilig ausschließlich aus dem Blickwinkel von Spielräumen innerhalb des Lohnarbeits-Kapital-Verhältnisses betrachtet.

E.A.: Man könnte dem natürlich entgegenhalten, daß der Staat am Ende des vorigen Jahrhunderts als imperialistischer Staat die Expansion des Kapitals nationalstaatlich organisiert hat, während heute die Expansion des Staates eben dadurch stattfindet, daß staatliche Grenzen im Zuge der Deregulierung abgebaut werden. Alle Regeln, die da dereguliert worden sind, stellen ja gewisserweise Grenzen dar, und die verschwinden, während die Rolle des Staates in jener Phase hauptsächlich darin bestand, die Grenzen hinauszuschieben und damit natürlich auch Konflikte zu provozieren bis hin zur militärischen Auseinandersetzung, wie wir wissen. Aber kommen wir noch einmal zurück zu den osteuropäischen Transformationsländern. Du hast die Aufgabe des Staates eben doppelt umschrieben als ökonomischer Regulator, so sage ich vereinfachend, und als demokratisches Institutionensystem, die sich beide als solche entwickeln müssen. Wie würdest Du denn unter diesem Aspekt betrachtet die Rolle des Staates in Transformationsgesellschaften begreifen? Wie können die Staaten, beziehungsweise Staat, Gesellschaft und Ökonomie als ein Komplex dahin kommen? Ich glaube, das ist auch die Frage, die die Theoretiker und Theoretikerinnen umtreibt, die sich mit Transformationsprozesssen beschäftigen, wenn sie in diesem Zusammenhang von Hybridformen sprechen, also von Mischformen, in die sehr Vieles eingeht, das in Reinform (etwa wie die Modernisierungstheorie unterstellt) eigentlich nicht realisierbar ist? Ist Osteuropa nicht gewissermaßen ein Experimentierfeld für die Herausbildung hybrider Formen des Kapitalismus?

J.H.: Ja, das glaube ich auch, und auch, daß es offensichtlich keine bereits überzeugenden theoretischen Konzepte zur Erfassung der Hybridität gibt. Es ist ja wichtig festzuhalten, daß die osteuropäischen Länder nicht nur aus ökonomischen Gründen zusammengebrochen sind; eine solche Unterstel- 
lung wäre jedenfalls eine sehr grobe Verballhornung der materialistischen Methode. Die erste Bewegung war ja der politische Widerstand gegen bestimmte politische Herrschaftsformen, die den Menschen immer weniger Bewegungsfreiheit ließen. Man kann diese Formen natürlich auch als aus der Not einer unzureichenden Ökonomie geboren ansehen. Nur bin ich im Zweifel darüber, ob sich das alles so glatt ökonomisch ableiten läßt, ob da nicht vielmehr überschüssige eigenständige Momente sowohl von politischer Unterdrückung als auch von politischem Widerstand entstanden sind. Wenn das so ist wie ich vermute, dann gibt es in der Tat zwei relativ selbständige Prozesse: die politische Emanzipation und den Versuch der ökonomischen Konstitution einer funktionierenden Wirtschaft jenseits des gescheiterten Sozialismus. Weil eine sichtbare und überzeugende Alternative nicht da war, hat sich der Übergang zur kapitalistischen Marktwirtschaft angeboten, wobei der Westen natürlich auch ein politisch durchsetzungsfähiger Anbieter war. Doch theoretisch zwingend war und ist dies nicht. Die faktische Entwicklung in Mittel- und Osteuropa ist wohl damit zu erklären, daß die Auffassung durchgesetzt wurde, der politische Prozeß der Demokratisierung erfordere sozusagen als ökonomischen Unterbau eine kapitalistische Marktwirtschaft. Diese müsse im Eiltempo herbeigeführt werden. Doch gerade das ist weder theoretisch zwingend noch tatsächlich machbar. So kam es in dem Maße, wie die Vermarktwirtschaftlichung politisch durchgezogen wurde, in einigen Ländern (vor allem in Rußland) zu nachgerade chaotischen, ja katastrophalen und aller Demokratisierung ins Gesicht schlagenden Entwicklungen. Der mafiotische Kapitalismus ist durchaus mit der Vertreibung vom Land durch Feuer und Schwert in der ursprünglichen Akkumulation vergleichbar. Die pure Gewalt bei der Herstellung kapitalistischer Verhältnisse spielt auch in einigen osteuropäischen Ländern eine Rolle. Ich finde es sehr wichtig, auch diese Dimension der Transformation zu sehen und nicht nur auf die freundlichen Seiten $\mathrm{zu}$ schauen. Ich möchte aber doch noch auf eine Differenz hinweisen, die wir bei der Einschätzung der Rolle staatlicher Grenzen haben. Ich habe nicht den Eindruck, daß der gegenwärtige Kapitalismus die staatlichen Grenzen wirklich beseitigt hat. Am ehesten ist das der Fall in Integrationsräumen wie der EU, und nicht einmal hier ist es wirklich vollendet. Die nationalstaatliche Handlungsfähigkeit des Staates im Sinne der international agierenden Konzerne hat in den letzten 10 Jahren enorm zugenommen. Was abgenommen hat, ist die Fähigkeit der Gewerkschaften und anderer sozialer Bewegungen, ihre Ansprüche und Interessen in diesem Nationalstaat gegenüber den Interessen des Kapitals und hier besonders des exportorientierten Großkapitals durchzusetzen. Ich glaube, der nationalstaatliche Hintergrund des großen international agierenden Kapitals ist nach wie vor ein wesentliches Merkmal des modernen Kapitalismus. Das war auch im Zeitalter des Imperialismus nicht so 
alter des Imperialismus nicht so verschieden wenn auch die Formen des damaligen Staates offener aggressiv waren.

E.A.: Damit bin ich nicht ganz einverstanden. Die Globalisierung am Ende des 20. Jahrhunderts scheint mir doch einen sehr großen Unterschied gegenüber dem Imperialismus des frühen 20. Jahrhunderts zu machen, einfach deshalb, weil in Zeiten der Globalisierung die Grenzen alle abgesenkt werden bzw. fallen: von den Zollgrenzen angefangen bis zu den Regulierungsschranken für internationale Kapitalbewegungen. Die einzigen Schranken, die auch nationalstaatlich relevant sind und sogar noch verstärkt worden sind, sind diejenigen gegen Migranten und Migrantinnen, d.h. die Globalisierung der Märkte findet nicht gleichmäßig für alle Märkte statt. Der Arbeitsmarkt ist noch national protegiert, doch Löhne und Arbeitsbedingungen stehen trotzdem unter dem Druck der globalen Konkurrenz wegen der Globalität und Bewegungsfreiheit des Kapitals. Aber lassen wir dieses Problem an dieser Stelle auf sich beruhen und gehen wir doch noch einmal zurück zu der von Dir hervorgehobenen Parallelität von Stamokaptheorie, Regulationstheorie, »social structure of accumulation «-Ansatz. Das Interessante daran war ja bei allen drei Ansätzen der Versuch, nicht nur den Kapitalismus zu beschreiben und Kategorien zu finden, mit denen wie bei Marx der Kapitalismus oder das Kapitalverhältnis entschlüsselt, begrifflich reproduziert werden kann, sondern auch Phasen der kapitalistischen Entwicklung historisch zu unterscheiden, also so etwas wie ein Verhältnis von allgemeiner und historischer Theorie zu konstruieren. Wie würdest Du dieses methodische Herangehen heute sehen? Meinst Du, daß dieses Bestand hat, muß man daran weiterarbeiten, oder sollte man es aufgeben? Gibt es den Kapitalismus gar nicht mehr, sondern nur noch den modernen Kapitalismus der Globalisierung und früher mal den Imperialismus, die Konkurrenz oder auch das Monopol, oder muß man an dieser methodischen Struktur der Analyse des Kapitalverhältnisses festhalten und diese möglicherweise auch auf die Tendenzen der Herausbildung eines osteuropäischen Hybridkapitalismus anwenden?

J.H.: Das ist eine interessante Frage, auf die es zwei Antworten gibt. Erstens ist es natürlich wichtig, nicht nur zu schauen, ob das Kapital noch Kapital ist, sondern in welcher konkreten Form es sich bewegt. Im historischen Verlauf macht der Kapitalismus ohne Zweifel verschiedene Formwandel durch. Man kann die Herausbildung charakteristischer Eigenschaften für bestimmte Phasen, etwa für den Imperialismus oder den Faschismus, herausarbeiten. Das wird man auch heute in einer Phase der sogenannten Globalisierung machen müssen, auch wenn man am Ende des vorigen Jahrhunderts bereits ähnliche Entwicklungen wie heute feststellen konnte. (Es gab beispielsweise, wie heute, keinerlei Grenzen für das international operierende Finanzkapital.) Das ist das eine. Die andere Antwort 
betrifft die Widerstandsformen gegen und die Alternativen zu einer bestimmten Form der kapitalistischen Entwicklung. Hier weist die StamokapTheorie Lücken auf, die aber auch die Regulationstheorie oder der »social structure of accumulation «-Ansatz nicht überzeugend gefüllt haben. Die Feststellung einer Phasenfolge der kapitalistischen Entwicklung wird immer dann problematisch, wenn sie dabei eine Art historischer Logik unterstellt: Man konstatiert bestimmte Eigenschaften, die eine Entwicklungsphase charakterisieren, nennt das Ensemble von Eigenschaften beispielsweise Fordismus und untersucht dann die Prozesse der Veränderung und Auflösung der Eigenschaften, bis dann eine neue Phase kommt, sozusagen eine andere Formation, der wir wieder einen fest umrissenen Namen geben. Ich bin im Zweifel, ob das ein produktives Herangehen ist. Demgegenüber halte ich es für sinnvoll, nach den vorherrschenden Bewegungsformen zu fragen und ihren Bedingungen, in der Nachkriegsphase also nach der Bedeutung des staatlichen Interventionismus, der Masseneinkommen und Massenproduktion. Warum und wie hat sich der Interventionismus erschöpft, welche neuen Widersprüche sind entstanden, welche neue Dynamik bildet sich heraus etc. Ich habe nichts dagegen, einen Phasenbegriff für eine Konstellation solcher Faktoren zu reservieren, die über einen gewissen Zeitraum relativ stabil ist, wie beispielsweise den Begriff des Fordismus. Allerdings halte ich den Begriff Postfordismus für außerodentlich unglücklich, weil er keine derartig stabile Konstellation anzeigt, und es diese vermutlich gegenwärtig auch gar nicht gibt. Da ist der »social structure of accumulation «-Ansatz offener: Akkumulation findet immer innerhalb bestimmter sozialer Strukturen statt, die bestimmte Ausformungen des Verhältnisses Lohnarbeit-Kapital (und anderer sozialer Verhältnisse) bestimmen. Veränderungen betreffen das Wechselspiel zwischen stärkerer Dominanz des Kapitals, stärkerer Dominanz der Arbeiterbewegung und anderer sozialer Kräfte als Resultat sozialer Konflikte. Daraus entstehen Konstellationen und Muster, in denen sich das allgemeine Kapitalverhältnis bewegt. Das halte ich für einen vernünftigen Ansatz. Wenn wir davon reden, daß sich in den letzten zwanzig Jahren der Neoliberalismus durchgesetzt hat, dann sind damit bestimmte Formen der Kombination von Politik und Ökonomie gemeint, die in dieser Zeit eine gewisse Dominanz erreicht haben und gegenwärtig bereits brüchig werden. Wollen wir das jetzt PostNeoliberalismus nennen oder uns zunächst damit bescheiden, die Brüchigkeit festzustellen und jene Ansatzpunkte zu benennen, an denen es sich lohnt, sich für politische Emanzipation und ökonomischen Fortschritt einzusetzen?

E.A.: Bevor wir an dieser Stelle weiterdiskutieren, und auf der theoretischmethodischen Ebene verbleiben, auf der wir uns mit der Sinnhaftigkeit einer Phaseneinteilung kapitalistischer Entwicklung und mit dem Verhältnis 
von Theorie und Geschichte befassen, eine andere Frage, die Du angesprochen hast. In bezug auf den Neoliberalismus könnte man ja folgende These aufstellen: In den sogenannten goldenen Jahren nach dem Zweiten Weltkrieg, in der Blütezeit des Fordismus - um diesen Begriff noch einmal zu verwenden - hat die keynesianische Politik - sagen wir abgekürzt: die Makroökonomie - das Sagen gehabt. Diese Makroökonomie mit Fiskalund Geldpolitik, mit Nachfragesteuerung und einer sehr aktiven Rolle des Staates also, ist vorbei und hat dem Primat der Mikroökonomie Platz gemacht. Die Vielfalt mikroökonomischer Entscheidungen soll, gesteuert durch den frei wirkenden Marktmechanismus, den Wohlstand optimieren. Inzwischen gibt es aber noch eine Vorstellung, vielleicht sogar ein anderes Paradigma der Wirtschaftspolitik, das sich auf die meso- und metaökonomische Ebene bezieht. Dieser Ansatz trägt gewissermaßen der Einbettung der Ökonomie in die Gesellschaft, also der »social structure of accumulation « Rechnung, obwohl das in dem Ansatz etwas anders angegangen worden ist als in den derzeit modernen mesoökonomischen Ansätzen einer Theorie der systemischen oder strukturellen Wettbewerbsfähigkeit. Die darin zum Ausdruck kommende systemische Herangehensweise überwindet ja nicht nur die Mikroökonomie, sondern auch die Makroökonomie. Diese werden nicht ausgeklammert, aber ihnen wird ein ganz neuer Stellenwert im politischen Konzept zubilligt. Meinst Du nicht, daß in diesen Politikkonzepten und in deren Wandel auch so etwas wie eine Phasenlogik enthalten ist?

J.H.: Wenn man die Frage solide beantworten will, muß man sich damit beschäftigen, wie der Übergang der keynesianischen Phase der »goldenen Jahre « in die neoliberale Phase eigentlich herbeigeführt worden ist. Der Keynesianismus war keineswegs ein makroökonomisches Projekt, sondern nach dem Zweiten Weltkrieg aufgrund eines bestimmten Kräfteverhältnisses zwischen Arbeit und Kapital vor allem ein politisches und soziales Projekt. Da spielten die Erfahrungen des Zweiten Weltkrieges und der Weltwirtschaftskrise hinein. Die Theorie des Staatsinterventionismus war sozusagen das Tüpfelchen auf dem i und dieser ausdrücklich als Instrument zur Erreichung von Vollbeschäftigung konzipiert.

E.A. ... also Keynes plus Beveridge...

J.H. Jawohl, genau. Dieses sozial-ökonomische Projekt ist denkbar schlecht mit dem Wort Gesellschaftsvertrag bezeichnet. Bei Gesellschaftsvertrag assoziiere ich immer, daß zwei Leute am Tisch sitzen und sich einigen. Der Keynesianismus war aber in erster Linie das Resultat von Auseinandersetzungen, in denen, darauf kommt es mir an, dem Kapital Zugeständnisse abgerungen worden sind. Mit diesen Zugeständnissen konnten die Unternehmen hinterher ganz gut leben. Dennoch wurden sie doch immer als Zugeständnisse empfunden. Ende der 60er, Anfang der 70er Jahre 
schlaffte bekanntlich aus Gründen, die ich hier nicht ausbreiten möchte, die Akkumulationsdynamik ab. Im Rückblick halte ich die 70er Jahre für eine ganz entscheidende historische Phase für die Entwicklung des Kapitalismus sowohl in der Bundesrepublik wie auch in anderen Ländern. Denn faktisch stand die Frage nach der Entwicklungsrichtung des Kapitalismus auf der Tagesordnung. Dabei stellten sich grundsätzlich zwei Alternativen: Entweder man treibt die Reformen weiter und geht zu einer Art Investitionslenkung über. Ich erinnere mich noch an die vielen Debatten über Investitionslenkung in dieser Zeit zu Beginn der 70er Jahre. Diese Debatten folgten im Grunde dem Gefühl, daß die Entwicklung in der bisher eingeschlagenen Reformrichtung nur weitergehen könne, wenn die wirtschaftspolitische Regulierung ausgeweitet und intensiviert wird. Oder man ist der Überzeugung, daß das nicht geht und von nun an der Weltmarkt das Gesetz diktiert. Für die erste Alternative wäre es eigentlich notwendig gewesen, eine schlüssige Konzeption auszuarbeiten und die politischen Kräfte zu ihrer Durchsetzung zu mobilisieren. Dies ist bekanntlich nicht gelungen. Die Folge war, daß das Kapital vieles »zurückbekommen« hat, was es nach dem Krieg aufgeben mußte. Eine enorme Umverteilung und ein ebenso enormer Deregulierungsprozeß setzten ein. Sie müssen m.E. als ein politischer Roll-Back-Prozeß aufgrund geänderter ökonomischer Verhältnisse und unzureichenden Widerstandes interpretiert werden. Noch einmal: die ökonomischen Verhältnisse haben diesen Roll-Back-Prozeß nicht determiniert. Die Alternative wurde weder konzeptionell noch politisch ergriffen. Heute geht es in meiner Sicht weniger um den Versuch, zwischen mikround makroökonomischer Ebene auf einer Mesoebene zu vermitteln. Eher geht es darum, den sozialen Rückschritt der vergangenen Jahrzehnte rückgängig, eine Konzeption für sozialen Fortschritt und Gerechtigkeit zu entwickeln und die aktuelle und zusätzliche Frage der ökologischen Nachhaltigkeit zu thematisieren. Ich sehe im Augenblick noch nicht, wie dies möglicherweise kollidiert oder konvergiert mit dem, was Du Mesoebene nennst. Vielleicht kannst Du das noch etwas zuspitzen?

E.A.: Ich beziehe mich mit dem Hinweis auf die Mesoebene auf die Theorien über »systemische Wettbewerbsfähigkeit«. Demnach hat die Makroökonomie für stabile Verhältnisse, stabilen Geldwert, Attraktivität der Währung usw., für den »Standort« im globalen Raum zu sorgen. Alle Faktoren der Wettbewerbsfähigkeit, die nicht auf der betrieblichen, also mikroökonomischen Ebene angebotspolitisch bereitgestellt werden können, von der Infrastruktur angefangen bis hin zur Vernetzung zwischen Wirtschaft und Gesellschaft, zwischen Unternehmen und Wissenschaft, zwischen Gewerkschaften und Arbeitgeberverbänden im System der industriellen Beziehung, können nur durch koordinierte gesellschaftliche und politische Aktion geschaffen werden. Das ist für die Kontinuität der Produktion 
und daher die Wettbewerbsfähigkeit des Standortes wichtig. Dieses wird in den Konzepten der systemischen Wettbewerbsfähigkeit, etwa in jenen des Deutschen Instituts für Entwicklungspolitik, mit der Metaebene in Zusammenhang gebracht. Man braucht Institutionen und Verfahren, die einen gesellschaftlichen Konsens erzeugen und stärken, den Habitus bestimmen etc. Ich halte diesen Ansatz für sehr idealistisch und normativ konstruriert. Wenn man analytisch herangeht, wird man nicht so viele Bedingungen vorfinden, wie benötigt werden, damit diese Ebenen auch alle funktionieren, d.h. daß die Wettbewerbsfähigkeit wirklich gesichert wird und gar steigt. Aber immerhin ist dies ein Konzept, das unter phasentheoretischen Aspekten die Betonung der Makroökonomie einerseits und der Mikroökonomie andererseits historisch überwindet. Mikro- und Makroökonomie ist ja immer noch die Unterteilung, die das Angebot der Wirtschaftstheorie an den Universitäten strukturiert. Da hat natürlich alles das, was meso-, meta- oder megaökonomisch, d.h. oberhalb der makroökonomischen Instanzen des Nationalstaates passiert, keinen Platz.

J.H.: Davon kann ich ein Liedchen singen, da ich gerade eine Einführungsvorlesung in die Makroökonomie halte und gezwungen bin, mich mit diesem Problem herumzuschlagen. Mit der Theorie der systemischen Wettbewerbsfähigkeit jedoch habe ich ein Problem obgleich sie ja den Gedanken der großen Komplexität und der Pfadabhängigkeit von Entwicklungen aufnimmt und damit auch eine Brücke zu der politischen Konzeption von Hegemonie schlägt, die für politische Handlungsfähigkeit entscheidend ist. Ich stimme Deiner Einschätzung des idealistischen Charakters der Theorie zu und möchte ihn durch die Kritik an der mangelnden Klarheit des Begriffes Wettbewerbsfähigkeit ergänzen. Bei diesem Begriff kommt man m. E. nicht um eine sehr klare konzeptionelle Festlegung herum: Bezeichnet man mit Wettbewerbsfähigkeit eines Landes die Fähigkeit seiner Wirtschaft, die eigene Bevölkerung mit Gütern und Dienstleistungen ordentlich zu versorgen, dann sollte man dies lieber Leistungsfähigkeit nennen - oder heißt Wettbewerbsfähigkeit die Errichtung bzw. der Ausbau einer Überlegenheitsposition auf dem Weltmarkt? Diese Frage beantwortet auch die Theorie von der systemischen Wettbwerbsfähigkeit nicht. Das Ziel Wettbewerbsfähigkeit als Überlegenheit auf dem Weltmarkt läuft auf eine Strategie hinaus, die nationale Kapitalverwertung auf Kosten anderer zu organisieren. Die angestrebte Überlegenheitsposition auf dem Weltmarkt impliziert immer auch ein Unterlegenheitsposition von anderen. Die Geschichte zeigt, daß dies auf Dauer nicht gutgehen kann. Daher möchte ich darauf insistieren, daß Wettbewerbsfähigkeit nur dann dauerhaft durchhaltbar ist, wenn sie in eine Form globaler wirtschaftspolitischer Kooperation eingebettet ist. Andere Gesellschaften in eine schlechtere Position zu drücken, ist als generelle Linie weder klug noch machbar. Internationale Arbeitsteilung 
ist wünschenswert. Ihre Herstellung erfordert aber nicht nur Konkurrenz, sondern auch Kooperation. Hier sind wir an einem Kernproblem der modernen Ökonomie. Einerseits muß ein gewisser Leistungsdruck durch Konkurrenz erhalten werden. Andererseits artet der Leistungsdruck, so wie er gegenwärtig organisiert ist, in eine hemmungslose und ruinöse Konkurrenz aus, die zur unendlichen Beschleunigung ökonomischer Prozesse in der Zeit, zur Ressourcenzerstörung, zur Krise und zur sozialen Polarisierung führen muß. Dies ist unvermeidbar, wenn es keine politischen Grenzen der Konkurrenz gibt. Dies ist die Grundlage für die Notwendigkeit ökonomisch-politischer Kooperation. Im Grunde war die langfristige Komplexplanung der sozialistischen Länder im Rahmen des COMECON eine vernünftige Idee; nur ist sie in einer völlig unvernünftigen Weise durchgeführt worden. Arbeitsteilung im Weltmaßstab kann nicht nur Ergebnis der Konkurrenz (des Freihandels) sein, sie setzt auch wirtschaftspolitische Kooperation voraus. Die Organisation einer global arbeitsteiligen Wirtschaft nicht gemäß den Regeln des Freihandels in der Konkurrenz, sondern unter Entwicklung und Nutzung von Beziehungen der Kooperation das wäre tatsächlich so etwas wie ein Paradigmenwechsel.

E.A.: In der Konkurrenz findet tatsächlich - um das Wort von Paul Krugman zu benutzen - eine Art Wettlauf der Besessenen statt. In dem alle Standards nach unten gedrückt werden: es handelt sich um the race to the bottom, es gibt nur Verlierer. Doch ist Kooperation wirklich eine Lösung? Man könnte boshaft werden und sagen: der Stamokap des Jahres 1998 ist die Kooperation Daimler/Chrysler, mit der die Großkonzerne versuchen, der Konkurrenz aus dem Wege zu gehen bzw. sie durch globale Kooperationsformen aufzuheben. Daimler und Chrysler befinden sich in guter Gesellschaft, denn auch viele andere Branchen- und Unternehmenskomplexe auf globaler Ebene sind gebildet worden. Das Jahr 1997 war das Jahr mit der höchsten Zahl von Unternehmenszusammenschlüssen weltweit. Die Neue Züricher Zeitung gibt den Wert der zusammengeschlossenen Unternehmen mit etwa 1.600 Milliarden US-Dollar an. Kooperation, um der Konkurrenz zu entgehen, ist also nicht billig. Hat das etwas mit Stamokap zu tun? Ist das die Kooperation, die Du meinst? Meine Frage ist polemisch aber ernsthaft. Denn wie muß man sich zu diesen Fragen theoretisch und dann natürlich auch politisch verhalten?

J.H.: Der Begriff der Kooperation ist ja von Marx sehr positiv eingeführt worden...

E.A.: ...Bei Marx ist Kooperation aber vor allem Kooperation auf betrieblicher Ebene.

J.H.: Ja, auf betrieblicher Ebene in dem Sinne, daß von drei Leuten, die vor einem großen Stein stehen, keiner allein, wohl aber die drei zusammen ihn aufheben können. Das ist Kooperation zur Erhöhung der betrieblichen 
Leistungsfähigkeit. Auf dem Markt gibt es aber auch Kooperation, um Vorteile in der Konkurrenz gegenüber Dritten zu organisieren. Dies ist Sinn und Absicht des Kartells. Die Binnenkonkurrenz wird aufgehoben, um die Konkurrenzposition nach außen zu verbessern. Man muß eben unterscheiden zwischen der Konkurrenz als solcher und der Konkurrenzposition, zwischen Wettbewerbsfähigkeit und Wettbewerb. Alle Arten von interner Kooperation, die produktivitätssteigernd sind, wirken nur dann nach außen nicht polarisierend, wenn sie eingebunden sind in einen kooperativen Gesamtrahmen, der nur politisch bestimmt werden kann. Der Markt kann sich in keinen Rahmen begeben, der Markt ist Konkurrenz pur. Für die regionale und nationale Ebene ist dies längst bekannt und in der jeweiligen Rechtsordnung umgesetzt. Es gilt aber auch und in besonderem Maße auf internationaler Ebene. Demgegenüber hat sich seit den 70er Jahren eine Politik durchgesetzt, die meint, es sei ihre Aufgabe, das nationale Kapital wettbewerbsfähig zu machen, um es auf dem Weltmarkt ohne Kooperation gegen andere nationale Kapitale operieren lassen zu können. Der Staat funktionierte dabei sozusagen als Instanz zur Munitionierung für nationale Kapitale. Diese Strategie ist mitverantwortlich für die Krise und zunehmende Chaotisierung der Weltwirtschaft.

E.A.: Noch einmal zurück zur Frage der Kooperation. Man kann gute Argumente dafür anführen, daß sie stattfindet, etwa auf westeuropäischer Ebene. Das Weißbuch der EU-Kommission von 1993 ist ein wunderbares Beispiel dafür, wie Kooperation von seiten der Europäischen Kommission oder von anderen Instanzen auf europäischer Ebene organisiert werden soll, um die Wettbewerbsfähigkeit in der sog. Triadenkonkurrenz zu verbessern. Osteuropa kommt da lediglich insofern vor, als die europäischen Netze der Kommunikations- und Transportinfrastruktur auch nach Osteuropa ausgedehnt werden sollen. Aber Osteuropa ist in dem Konzept der EU-Kooperation nicht mitgedacht. Die Frage lautet schlicht, ob man sich in kapitalistischen Verhältnissen Kooperation so, wie Du es dargestellt hast, anders denken kann, denn als eine Waffe in der Konkurrenz, um stärker zu sein als die anderen, und um sie zu schwächen. Was können die anderen in solcher Lage, wenn durch Kooperation starke Blöcke wirtschaftlicher und politischer Macht entstehen, noch tun?

J.H.: Wenn man von der Logik des Einzelkapitals ausgeht, kann man gar nichts machen. Allerdings ist der gegenwärtige Kapitalismus ein relativ komplexes, auch politisches System. Die Dominanz des neoliberalen und mikroökonomischen Denkens hat ja inzwischen Gegenbewegungen hervorgerufen. In einer solchen Situation kann man einen Grundsatz aufstellen: Kooperation ist immer dann sinnvoll, wenn dadurch die Leistungsfähigkeit erhöht wird, ohne daß gleichzeitig Überlegenheitspositionen aufgebaut werden. Die EU ist gegenwärtig dabei, ihre Überlegenheitsposition 
gegenüber Osteuropa zu stabilisieren. Meines Erachtens kann das nicht gutgehen, es wird auch politisch destabilisierend wirken. Der Begriff der Kooperation ist für mich noch immer positiv besetzt; allerdings sehe ich die Gefahren: die imperialistische Kooperation, die Stamokap-Kooperation oder die europäische Kooperation gegen Drittländer. Dazu gehört auch der Euro als Ausdruck monetärer Kooperation gegen den Dollar. Internationale Kooperation erfordert ein politisch gewolltes und hergestelltes Gleichgewicht nach außen.

E.A.: Ich habe noch zwei Fragenkomplexe, die ich gern ansprechen wollte. Der erste geht noch mal zurück auf die Frage des politischen Kapitalismus in Osteuropa. Was kann man mit diesem Begriff anfangen im Zusammenhang mit der Diskussion über den Stamokap? Noch einmal zu der Phaseneinteilung, die wir ja schon diskutiert haben. Diese Phasen gehen auseinander hervor, bauen aufeinander auf, sie kommen immer dann zur historischen Geltung, wenn in einer Krise offensichtlich wird, daß es so wie bisher nicht mehr weitergeht. Neue Produktionsweisen oder Regulationsweisen sind immer auch Ausdruck davon, daß eine andere Regulationsweise an eine historische Grenze gestoßen ist. Die Frage ist also die nach den Krisenprozessen, die mit den Phasen zu tun haben. Wir leben ja momentan in der vielleicht tiefsten Krise des Kapitalismus überhaupt in seiner langen Geschichte. Das kann man schwer heute entscheiden, aber möglicherweise ist es so. Wie schätzt Du diese Krisenprozesse ein? Haben sie eine transformatorische Bedeutung einerseits für die osteuropäischen Länder, die gerade die Transformation von 1989 begonnen haben, aber auch für die sich selbstsicher fühlenden westlichen kapitalistischen Gesellschaften? Steckt da so etwas wie ein transformatorisches Potential in diesem Krisenprozeß? J.H.: Die transformatorische Bedeutung von Krisen setzt sich aus zwei sehr unterschiedlichen Elementen zusammen. Einerseits haben wir die Reaktion des Kapitals auf die Tatsache, daß die Verwertung nur mit zunehmenden Widersprüchen und Schwierigkeiten so weiter betrieben werden kann wie bisher. Auf der anderen Seite entstehen soziale Bewegungen. Sie sind Ausdruck dessen, daß die von den Strategien des Kapitals betroffenen Menschen nicht mehr bereit sind, deren Folgen so über sich ergehen zu lassen wie bisher. Es kommt nun sehr darauf an, wie das Verhältnis zwischen diesen beiden Elementen sich entwickelt. Ich bin skeptisch gegenüber dem Begriff des lernenden Kapitals, das in die Krise kommt und dann von sich aus etwas anderes überlegt. Lernprozesse finden nur unter dem Druck der sozialen Basis statt. Bestimmte Arbeitszeitmodelle, die Monotonie des Arbeitsprozesses etc. stoßen zunehmend auf Ablehnung und Widerstand. Beide Elemente gemeinsam bilden so etwas wie ein Transformationspotential. Dessen Verwirklichung und Dynamik hängt von der Gewichtung der Elemente ab. Handelt es sich um eine kooperative Einbindung der sozialen 
Basis, deren Wünsche in bescheidenem Maße berücksichtigt werden, in ein ansonsten durch die Interessen des Kapitals bestimmtes Entwicklungsmuster - oder handelt es sich um einen sehr schmerzhaften, unter heftigen sozialen Bewegungen herbeigeführten Richtungswechsel der sozialökonomischen Entwicklung? Wenn man ganz optimistisch ist, könnte man die sozialen Kämpfen in Frankreich 1997 und die relativ starken Paukenschläge in der französischen Wirtschaftspolitik als einen Auftakt für einen Richtungswechsel interpretieren, der im Kern - wenn auch gegenwärtig nur in Ansätzen sichtbar - transformatorische Bedeutung hat, weil ja beispielsweise auf ganz neue Arbeitszeitmodelle, auf eine Aufwertung binnenwirtschaftlicher Reproduktionsprozesse gesetzt wird. Das wäre schon deshalb eine erhebliche Transformation, weil ein größerer Teil der binnenwirtschaftlichen Produktionskapazität nicht mehr auf zu exportierende Autos gerichtet sein könnte, sondern auf in der Binnenwirtschaft abzusetzende Güter, die im Zweifelsfalle über den einfachen Massenkonsum hinausgehen. Aber dieses würde nur geschehen können, weil die Lohnansprüche oder die Arbeitszeitansprüche der Beschäftigten gegenüber den Unternehmen durchgesetzt werden und sich herausstellt, daß letztere entgegen ihren bisherigen Beteuerungen damit durchaus leben können.

E.A.: Die Ansätze zum politischen Kapitalismus sind nach meinem Dafürhalten deshalb so interessant, weil sie den Versuch machen, die vielen Aspekte und Dimensionen der Transformationprozesse, die manchmal sehr überraschend sind, im Zusammenhang zu sehen. Transformation ist ein politischer Prozeß und nicht nur ökonomische Anpassung an Marktgegebenheiten durch einen big bang und dergleichen. In diesen Theorien kommen allerdings die sozialen Bewegungen, von denen Du gesprochen hast, als Auslöser der transformatorischen Potenz der Krise oder auch der Phasenüberwindung zu kurz. Es müssen also die Massen, jetzt ganz abstrakt gesprochen, hinter den Transformationsprozessen stehen. Da hat ja die Stamokap-Theorie ihre ganz konkreten Vorstellungen. Antimonopolistische Bündnisse sollten es sein, um die führende Organisation der Arbeiterklasse herum. Heute sind wir da alle etwas vorsichtiger und auch weniger spezifisch und sagen, es ist die Zivilgesellschaft, die sich da bewegen muß. Manche sagen dann wieder etwas spezifischer: es sind die Nichtregierungsorganisationen, die neuen sozialen Bewegungen. Natürlich spielen auch die Arbeiter und ihre Gewerkschaften noch eine gewisse Rolle, aber nicht mehr eine zentrale, jedenfalls sind sie nicht der Kern eines antimonopolistischen Bündnisses. Wie das in Osteuropa aussieht, sagen die TheoretikerInnen zum politischen Kapitalismus eigentlich nicht, das wäre noch genauer zu eruieren. Aber wie muß man heute die Frage nach den sozialen Bewegungen im Westen einschätzen? War es so, wie die Stamokap-Theorie das gemacht hat, damals richtig und heute falsch, oder war es damals schon 
falsch? Wie ist die Frage der Zivilgesellschaft, der Nichtregierungsorganisationen, der neuen sozialen Bewegungen in diesem Kontext einzuschätzen?

J.H.: Das Konzept des antimonopolistischen Bündnisses in der StamokapTheorie hatte einen starken Punkt: die Bestimmung des Hauptgegners. Staatliche Politik wird im hohen Maße bestimmt durch Einflußnahme starker Kapitalgruppen. Das ist auch heute noch so. Insofern ist es wichtig, danach zu fragen, wo eigentlich das Zentrum der Macht liegt, die uns so viel zu schaffen macht. Andererseits war das Konzept des antimonopolistischen Bündnisses in zwei Punkten problematisch. Erstens enthielt es einen Führungsanspruch, nicht nur der Arbeiterklasse sondern der Partei der Arbeiterklasse. Dieser Führungsanspruch war weder theoretisch gerechtfertigt, damals nicht und heute auch nicht, noch machte er politisch Sinn, da er sowieso nicht durchgesetzt werden konnte. Zweitens war die Kette der Abstufungen von Bündnisschichten gemäß dem Abstand zum Kern der Arbeiterklasse, der immer noch definiert war als Industriearbeiterschaft, auch schon damals nicht haltbar. Wenn wir heute über Bündnisse nachdenken, geht es darum, soziale Bewegungen in ihrer ganzen Breite einzubeziehen, genauer gesagt demokratische soziale Bewegungen, denn es gibt ja auch soziale Bewegungen, die weniger demokratisch sind. Ich bin aber insofern Traditionalist, als ich der Arbeiterbewegung nach wie vor eine zentrale Bedeutung zumesse. Daher sind aus meiner Sicht nach wie vor die Gewerkschaften als Organisationen der Arbeiterbewegung nicht nur wegen ihrer Tradition, sondern auch wegen ihrer aktuellen Position im Reproduktionsprozeß und als soziale Bewegung ganz zentral. Eine Mobilisierung der Gewerkschaften für einen Neuanfang in der Wirtschafts- und Sozialpolitik ist daher ganz außerordentlich wichtig.

E.A.: Kann man, um es erneut etwas polemisch zuzuspitzen, vielleicht folgendes sagen: Die Stamokap-Theorie und die Bündnisstrukturen, die sich daraus ergeben haben und sich auch zum Teil heute noch ergeben, hatten etwas sehr Ordentliches vor Augen, während heute die politischen Subjekte etwas außerordentlich Chaotisches sind: Die vielen neuen sozialen Bewegungen, die Frauen, die dazwischenfunken, die Gewerkschaften, die sicherlich auch existieren aber heute eine ganz andere Rolle spielen als damals. Ist es nicht vielleicht ein Ausdruck der Unübersichtlichkeit der Verhältnisse, die die theoretischen Veränderungen andeuten? Die Frage lautet dann, ob es überhaupt möglich ist, wieder Ordnung ins Kategoriensystem zu bringen?

J.H.: Das sind zwei unterschiedliche Fragen. Ob und wann man wieder Ordnung in die theoretischen Ansätze und Konzepte bringen kann, wissen wir jetzt noch nicht. Ist es wünschenswert, wieder Übersichtlichkeit zu schaffen, das ist die andere Frage. Ich beantworte sie mit ja, weil ich Un- 
übersichtlichkeit nicht für ein Ziel halte. Die Stamokap-Theorie hat in einer ersten Phase alles ein- und unterordnen wollen, was unübersichtlich war und nicht so recht ins Konzept paßte. Das ist zu kritisieren und hat ja auch nicht funktioniert. In einer zweiten Phase hat sie mit großer Geste gesagt, wir öffnen uns für alle, anstatt zur Kenntnis zu nehmen, daß wir ein Teil von allen sind. Das ist kein kleiner Unterschied. Diese letzte Position ist das, was eigentlich ansteht und hilfreich sein kann bei der Analyse aktuellen Entwicklungen. Ich gehe davon aus, daß einerseits die gegenwärtige theoretische und politische Unübersichtlichkeit eine Reflexion der unübersichtlichen objektiven Verhältnisse ist und insofern auch nicht vorschnell in Schemata zu pressen ist, daß es aber andererseits wünschenswert wäre, eine neue Übersicht zu gewinnen über das, was vor sich geht. Dies erscheint mir auch erstrebenswert unter dem Aspekt politischer Handlungsfähigkeit, die sich nicht in Abwehr und Widerstand erschöpft, sondern auch Vorstellungen über die Ziele politischen Handelns entwickelt. Dabei werden wir unser Kategoriensystem sicher erweitern, möglicherweise verändern müssen. Zur Zeit habe ich allerdings auch den Eindruck, daß manche Kategorien marxistischer Theorie und auch manche Konzepte der Stamokap-Theorie vor dem Vergessen gerettet und manche wieder in Erinnerung gerufen werden müssen.

\section{Literatur (zu den angesprochenen AutorInnen und Themen)}

Aglietta, Michel (1979): A Theory of Capitalist Regulation. The US Experience, London.

Altvater, Elmar (1975): Wertgesetz und Monopolmacht, in: Zur Theorie des Monopols. Staat und Monopole, Argument Sonderbände 6, Berlin, S.129-198.

Autorenkollektiv (Leitung: N.N.Inosemzew) (1972): Politische Ökonomie des heutigen Monopolkapitalismus, Berlin (Übersetzung aus dem Russischen).

Autorenkollektiv des Instituts für Gesellschaftswissenschaften beim ZK der SED (Leitung: Otto Reinhold) (1971): Der Imperialismus der BRD, Berlin.

Boccara, Paul (1976): Studien über den staatsmonopolistischen Kapitalismus, seine Krise und seine Überwindung, Frankfurt/M. (Übersetzung aus dem Französischen).

Bowles, Samuel; Gordon, David M.; Weisskopf (1983): Beyond the Waste Land, New York.

- (1986): Power and Profits. The Social Structure of Accumulation and the Profitability of the Postwar U.S. Economy, in: Review of Radical Political Economics, vol 18, no 1\&2, S.132167.

Burawoy, Michael; Krotov, Pavel (1992): Der Übergang vom Sozialismus zum Kapitalismus in der früheren Sowjetunion, in: PROKLA 89, S.508-551.

Demirovic, Alex; Krebs, Hans-Peter, Sablowski, Thomas (Hrsg.) (1992): Hegemonie und Staat. Kapitalistische Regulation als Projekt und Prozess, Münster.

Dolata, Ulrich; Huffschmid, Jörg (1992): Deterministische Phasentheorie und unterkomplexes Verflechtungsmodell. Überlegungen zur weiteren Handhabung der Theorie des staatsmonopolistischen Kapitalismus, in: Z. Zeitschrift Marxistische Erneuerung Nr.10, S.61-75.

Ebbighausen, Rolf (Hrsg.) (1974): Monopol und Staat. Zur Marx-Rezeption in der Theorie des staatsmonopolistischen Kapitalismus, Frankfurt/M.

Gündel, Rudi; Heininger, Horst; Hess, Peter; Zieschang, Kurt (1967): Zur Theorie des staatsmonopolistischen Kapitalismus, Berlin

Heininger, Horst (1997): Zur Aktualität der Theorie des staatsmonopolistischen Kapitalismus, in: Z. Zeitschrift Marxistische Erneuerung Nr. 31, S.45-68. 
Hirsch, Joachim (1995): Der nationale Wettbewerbsstaat. Staat, Demokratie und Politik im globalen Kapitalismus, Berlin/Amsterdam.

Hübner, Kurt (1989): Theorie der Regulation. Eine Kritische Rekonstruktion eines neuen Ansatzes der Politischen Ökonomie, Berlin.

Huffschmid, Jörg (1975): Begründung und Bedeutung des Monopolbegriffs in der marxistischen politischen Ökonomie, in: Zur Theorie des Monopols. Staat und Monopole, Argument Sonderbände 6, Berlin, S.4-92.

Jung, Heinz (1978): Die privatmonopolistische Entwicklungsvariante des staatsmonopolistischen Kapitalismus in der BRD: Voraussetzungen, Inhalt, Perspektiven. Entwicklungstendenzen 1973 bis 1978, in: Marxistische Studien. Jahrbuch des IMSF Nr. 1, S.9-57.

Jung, Heinz; Schleifstein, Josef (1979): Die Theorie des staatsmonopolistischen Kapitalismus und ihre Kritiker. Eine allgemeinverständliche Antwort, Frankfurt/M.

Katzenstein, Robert (1973): Zur Theorie des staatsmonopolistischen Kapitalismus, in: PROKLA 8/9, S.1-16.

- (1975): Zur Frage des Monopols, des Monopolprofits und der Durchsetzung des Wertgesetzes im Monopolkapitalismus, in: Zur Theorie des Monopols. Staat und Monopole, Argument Sonderbände 6, Berlin, S.93-128

Lipietz, Alain (1985): Akkumulation. Krisen und Auswege aus der Krise. Einige methodische Überlegungen zum Begriff der »Regulation«, in: PROKLA 58, S.109-138.

Mahnkopf, Birgit (Hrsg.) (1988): Der gewendete Kapitalismus. Kritische Beiträge zur Theorie der Regulation, Münster.

Scherrer, Christoph (1988): Der »Social Structure of Accumulation«-Ansatz: Ein Interpretationsmodell des Aufstiegs und Niedergangs der US-Ökonomie, in: PROKLA 73, S.131-148.

Staniszkis, Jadwiga (1998): Postkommunismus. Versuch einer soziologischen Analyse, in: PROKLA 112, S. 375-394.

Stark, David (1994): Nicht nach Design: Rekombiniertes Eigentum im osteuropäischen Kapitalismus, in: PROKLA 94, S. 127-142.

Tatur, Melanie (1998): Ökonomische Transformation, Staat und moralische Ressourcen in den post-sozialistischen Gesellschaften, in: PROKLA 112, S.339-374.

Wirth, Margaret (1973): Zur Kritik der Theorie des staatsmonopolistischen Kapitalismus, in: PROKLA 8/9, S.17-46. 